\title{
NIVEL ALTO DEL ARSÉNICO Y SU REPERCUSIÓN EN LA SALUD *
}

\author{
BHIGH LEVEL OF ARSENIC AND ITS IMPACT ON HEALTH
}

\author{
${ }^{1}$ Darwin Wiliams Enriquez Castro
}

\begin{abstract}
RESUMEN
El arsénico se encuentra ampliamente distribuido en la corteza terrestre. Está presente en rocas, suelos, agua y aire. Su mayor amenaza para la salud pública reside en la utilización de agua contaminada para beber, preparar alimentos y regar cultivos alimentarios de manera crónica pudiendo causar cáncer, lesiones cutáneas, problemas de desarrollo, enfermedades cardiovasculares, neurotoxicidad y diabetes. Según la OMS, los niveles de arsénico en el agua potable no deben exceder los $0,01 \mathrm{mg} / \mathrm{L}$. En el presente trabajo que es descriptivo, transversal, que se realizó recolectando datos a nivel local, regional y nacional, e internacional. Se observó que en la localidad de Tacna hay incrementados niveles de arsénico en promedio es de $0,056 \mathrm{mg} / \mathrm{dL}$, que supone estar hasta cuatro veces lo normal en los caños de agua potable en diferentes distritos. Además hay un incremento del tipo de cáncer que está relacionado con los originados por el arsénico como son los canceres de Piel, estomago, vesícula, entre otras. Se recomienda Instalar sistemas de eliminación del arsénico ya que la ciudad de Tacna por ser zona volcánica tiene alto nivel de arsénico. Otra opción puede ser recolectar agua con bajos niveles de arsénico provisoriamente.
\end{abstract}

Palabras clave: Arsénico, agua potable, salud.

ABSTRACT

Arsenic is widely distributed in the earth's crust. It is present in rocks, soil, water and air. His biggest threat to public health is the use of contaminated water for drinking, food preparation and water chronically food crops can cause cancer, skin lesions, developmental problems, cardiovascular disease, neurotoxicity and diabetes. According to OMS, arsenic levels in drinking water should not exceed 0,01 $\mathrm{mg} / \mathrm{L}$. In the present work is descriptive, transversal, conducted by collecting data at local, regional and national level, and international. It was noted that in the town of Tacna there are increased levels of arsenic average is $0,056 \mathrm{mg} / \mathrm{dL}$, which means being up to four times normal in water pipes in different districts. There is al so an increasing type of cancer that is associated with those caused by arsenic as are skin cancers, stomach, bladder, among others. Install recommended arsenic removal systems as the city of Tacna to be volcanic area it has a high level of arsenic. Another option may be collecting water with low levels of arsenic provisionally.

Keywords: Arsenic, drinking water, health.

\section{INTRODUCCION}

Fl arsénico es un metal que en nuestro medio presenta un grave problema en la salud pública pues se encuentra en un notorio elevado nivel por encima de lo permitido por la OMS. Según los estándares de la OMS, los niveles de arsénico en cl agua potable no deben exceder los $0,01 \mathrm{mg}$ por litro, en nuestro país también dado como dato en el 2011 (D.S. Nº31-2010-SA).

El arsénico está presente de forma natural en niveles altos en las aguas subterráneas de varios países.

$\mathrm{El}$ arsénico se encuentra ampliamente distribuido en la corteza terrestre con una concentración media de 2 $\mathrm{mg} / \mathrm{kg}$. Fstá presente en cantidades ínfimas en todo tipo de rocas, suclos, agua y airc. Los compuestos del arsénico varian en su toxicidad para los mamíferos de acuerdo a su estado de valencia, la forma química (inorgánico u orgánico), el estado físico (gas, solución, o polvo) y factores como la solubilidad, tamaño de la partícula, vclocidad de absorción y climinación, y presencia de impurezas. Fl consumo de arsénico ( $A$ s) en aguas de bebida durante largos periodos de tiempo se ha asociado a una enfermedad denominada Hidroarsenicismo Crónico Regional Fndémico (HACRF), que se caracteriza por presentar lesiones en picl y alteraciones sistémicas cancerosas y no cancerosas.

El arsénico existe en 4 estados de valencia: As (-III) As (0) (arsénico metaloide, estado de oxidación 0) As (III) (estado trivalente, arsenitos) As (V) (estado pentavalente, arseniatos). El arsénico es muy tóxico en su forma inorgánica.

Su mayor amenaza para la salud pública reside en la utilización de agua contaminada para beber, preparar alimentos y regar cultivos alimentarios.

La exposición prolongada al arsénico a través del

'Interno de Medicina Humana de la Facultad de Ciencias de la Salud de la Universidad Nacional Jorge Basadre Grohmann. Tacna- Perú.

* Trabajo de investigación ganador del premio "Daniel Alcides Carrión", como mejor trabajo de investigación a nivel estudiantil, organizado por Academia Nacional de Investigadores Médicos - ANDIMED, otorgado en el Congreso Internacional de Investigación Médica y II Congreso Nacional de Investigación Médica celebrado en Lima - Colegio Médico de Lima. 2014. 
consumo de agua y alimentos contaminados puede causar cáncer y lesiones cutáncas. También sc ha asociado a problemas de desarrollo, enfermedades cardiovasculares, neurotoxicidad y diabetes. ${ }^{3,45}$

La intervención más importante en las comunidades afectadas consiste en prevenir que se prolongue la cxposición al arsénico implantando un sistcma seguro de abastecimiento de agua potable.

El arsénico representa una amenaza importante para la salud pública cuando se encuentra en aguas subterráneas contaminadas. F.l arsénico inorgánico está naturalmente presente en altos niveles en las aguas subterráneas de diversos paises, entre ellos la Argentina, Bangladesh, Chile, China, la India, México y los Estados Unidos de América. Las principales fuentes de exposición son: cl agua destinada a consumo humano, los cultivos regados con agua contaminada y los alimentos preparados con agua contaminada. ${ }^{1}$

En 'Tacna 'Ticona y Cols, realizaron un estudio en la cual en contraron que el arsénico en cl agua potable superaba hasta 4 vces el limite superio la concentración media era de $54,5 \mathrm{ug} / \mathrm{l}$. Fl $56 \%$ de las muestras superó el umbral de Bangladesh de $50 \mathrm{ug} / \mathrm{L}$.

En otros distritos del pais como Juliaca y Caracoto, en el $96 \%$ de las muestras de agua subterránea la concentración de arsénico superaba el límitc cstablecido por la OMS. Asimismo, todas las muestras de agua recogidas en la sección del río Rímac, que atraviesa Lima, tenían concentraciones de arsénico superiores al limite de la OMS. $\Lambda 1$ validarlo en comparación con los valores de laboratorio, el kit EQ identificó de forma correcta contaminación por arsénico respecto al límite en el 95\% (106/111) de las aguas subterráneas y en el $68 \%(19 / 28)$ de las muestras de agua superficiales.

Por tener impacto a nivel nacional, regional y local, es un trabajo de investigación ético pues no se irrumpe con los derechos de personas, es actual en el ámbito de las investigaciones médicas en la localidad.

\section{MATERIAL Y MÉTODOS}

Es un trabajo descriptivo, transversal, que se realizó recolectando datos a nivel local, regional y nacional, e internacional.

Entre las tecnologias que permiten eliminar el arsénico destacan la oxidación, la coagulación-precipitación, la absorción, el intercambio de iones y diversas técnicas de membranas. Existe un número cada vez mayor de opciones cficaces y económicas para eliminar el arsénico en las fuentes de abastecimicnto de agua a pequeña escala o de tipo doméstico.

\section{RESULTADOS}

Se observó que en la localidad de Tacna hay incrementados niveles de arsénico en promedio es de 0,056 $\mathrm{mg} / \mathrm{dL}$, que supone estar hasta cuatro veces por encima del nivel permitido por la OMS.
Se observó que en varios distritos del Perú, también se muestra un incremento del nivel del arsénico en el agua potable, lo cual se complementara haciendo más trabajos de investigación.

Se observó que hay un incremento del tipo de cáncer que está relacionado con los originados por el arsénico como son los canceres de Piel, estomago, vesícula, cntre otras.

Hay múltiples estudios que muestran un relación entre el nivel de arsénico superior a $\operatorname{los} 0,05 \mathrm{mg}$ de arsénico por litro de a desarrollar cáncer.

\section{RECOMENDACIONES}

Se recomienda Instalar sistemas de eliminación del arsćnico ya que la ciudad de Tacna por ser zona volcánica tiene alto nivel de arsénico, ya sea de manera centralizada o a nivel doméstico.

Sustituir las fucntes de abastecimiento con clevados niveles de arsénico, por ejemplo aguas subterráneas, por fuentes de abastecimiento con bajos niveles de arsénico y microbiológicamente seguras, por ejemplo agua de lluvia o aguas superficiales debidamente tratadas. Otra opción pucde ser recolectar agua con bajos niveles de arsénico provisoriamente mientras utilizan el agua con alto nivel de arsénico para lavar ropa y bañarse.

Crear una comisión que tenga como meta disminuir el nivel del arsénico al evaluarlo periódicamente en el agua potable.

\section{REFERENCIAS BIBLIOGRÁFICAS}

1. Ministerio de Salud del Perú, Reglamento de la calidad del agua para el consumo humano, Decreto Supremo $\mathrm{N}^{\circ} 031$ 2010-SA, 2011.

2. Ministerio de Salud de la Nación, Hidroarsenicismo Crónico Regional Endémico HACRE: Módulo de Capacitación. Garcia, Susana Isabel - 1a ed. - Buenos Nires. Programa Nacional de Prevención y Control de las Intoxicaciones., 2011.

3. Chen, Y., \& Ahsan, H. (2004). Cancer burden from arsenic in drinking water in Bangladesh. American Journal of Public Health, 94(5), 741-744.

4. Chen, Y., Graziano, J. H., Parvez, F., Liu, M., Slavkovich, V., Kalra, T., ... Ahsan, H. (2011). Arsenic exposure from drinking water and mortality from cardiovascular disease in Bangladesh: prospective cohort study: BMJ : British Medical Journal, 342, d2431. doi:10.1136/bmj.d2431

5. Wasserman, G. A., Liu, X., Parvez, F., Ahsan, H., FactorLitvak, P., Kline, J., ... Graziano, J. H. (2007). Water Arsenic Exposure and Intellectual Function in 6-YearOld Children in Araihazar, Bangladesh. Emvironmental Health Perspectives, 115(2), 285-289.doi:10.1289/ehp.9501

6. Ticona, M. Contaminación del agua potable con arsénico y frecuencia del cáncer en la ciudad de Tacna 2010-2011.

7. Organización Mundial de la Salud, Fxposición al arsénico en el agua potable: una gran amenaza inadvertida para la salud en Perú, 2014.

\section{Correspondencia:}

Darwin Williams Enriquez Castro: wiliamsenriquezc@gmail.com
Fecha de Recepción: 10/03/2015

Fecha de Aceptacion: 01/06/2015 


\section{NORMAS EDITORIALES Y GUIA DE PRESENTACIÓN DE ARTICULOS CIENTÍFICOS PARA LA REVISTA CIENCIA \& DESARROLLO}

I a Revista Científica Ciencia \& Desarrollo pone a consideración de la comunidad científica y académica, sus normas editoriales y guía de presentación de los articulos científicos, con la finalidad de lograr que la publicación de la revista sea un espacio abierto para la divulgación de nuevos conocimientos.

\section{CRITERIOS DE PUBLICACIÓN, CITACIÓN Y REPRODUCCIÓN}

La revista Ciencia \& Desarrollo es una publicación semestral de caräcter cientifico teenológico, orientada a promover y difundir la investigación cn campos multidisciplinarios. Su publicación es impresa y en idioma español.

Para la publicación en la revista Ciencia \& Desarrollo los articulos presentados deben ser inéditos y pueden ser: artículo cientíico original, artículo de revisión, carta al editor, reporte de caso clínico, artículo de opinión, reseña de libro, ensayos, articulo breve. Los artículos publicados en esta revista pueden ser citados en otros documentos, siempre y cuando se indique la siguiente información: Revista Científica Ciencia \& Desarrollo. Tacna (Perú): Universidad Nacional Jorge Basadre Grohmann, número de la revista, página(s) y ano de publicación. ISSN 2304-8891. Si la reproducción de artículos publicados en la revista, es con fines académicos y sin ánimo de lucro, puede realizarse si se incluye la información establecida en el párrafo anterior. Si su reproducción implica otros usos, debe solicitarsc autorización escrita al Director de la Revista cientifica Cícncia \& Desarrollo.

La revista señala que la publicación de arúculos no da derecho a remuncración alguna y que la responsabilidad del contenido de los artículos es de los autores, inclusive en lo relacionado con la propiedad intelectual de otros autores $\mathrm{y} / \mathrm{o}$ fucntes.

La revista cientifica Ciencia \& Desarrollo recibe artículos de las ciencias básicas y aplicadas, tecnológicas y de ingenieria.

\section{TIPOS DE ARTÍCULOS}

2.1. Artículo científico original: La extensión del desarrollo no debe ser más de 12 páginas o caras. La redacción es en tercera persona. Dado que la finalidad de un artículo cientifico es comunicar los resultados de investigaciones, ideas y debates de una manera clara, concisa y fidedigna, se tomará en cuenta los principios fundamentales de la redacción científica: precisión, claridad y brevedad. Además de los compronentes del artículo científico deben cumplir con las siguientes caracteristicas.

Su estructura cs del siguiente tipo

Título: Debe contener en esencia el problema que cstá planteado, ser claro y preciso. Consta de 10 a 15 palabras, en caso de ser muy largo utilizar un subtitulo conciso. Su redacción es en mayúscula, respetando la redacción de las denominaciones de nombres cientificos.

Title: Es el título traducido correctamente en idioma inglés.

Autor/es: Si el trabajo ha sido realizado en equipo, debe colocarse como primer autor, el que tuvo mavor responsabilidad en la realización del trabajo.

Afiliación. Institución a la cual pertenecen los autores (especificar departamento o área por cada autor)

Resumen: Debe contener en forma escueta los objetivos, planteamiento del problema, materiales y métodos, y resultados. Fil resumen y abstract se redactan en un promedio de 200 a 250 palabras. Se redacta en ticmpo pasado. Debe ser escrito en un solo párrafo, se parando las oraciones con punto seguido. Sólo las palabras clave deben ir como punto aparte.

Palabras clave. No deben ser menores a dos ni mavores a cinco, ordenadas alfabéticamente.

Abstract. Es el resumen con una correcta traducción al inglés.

Key Word. Palabras clave correctamente traducidas al ingles.

Introducción: $\mathrm{E}$ lautor debe procurar que todo el trabajo forme una unidad desde el inicio hasta el final, coherente y relacionado. En esta parte se expone el problema, se informa lo que se conoce del mismo, se hará una revisión bibliográfica pertinente, se discute el objetivo general, fin principal y la hipótesis (si lo hubiera).

Material y Métodos: Descripción de los recursos humanos, materiales físicos, financieros y técnico administrativos empleados; explicación detallada del método empleado, señalando las técnicas estadísticas, si se utilizaron.

Resultados: Se expondrá en forma clara siguiendo una sccuencia lógica. Se exponen mediante figuras o tablas, donde se exponen la información resumida que confirman o no la(s) hipótesis y objecivos específicos planteados en la investigación. Deben limitarse a describir los propios hallazgos encontrados, evitando adelantar interpretaciones o comparaciones. Se redacta en pasado.

Discusión: En esta parte el autor podrá comparar, discutir, argumentar sus resultados con el de otros autores. Tomar en cuenta que los resultados se exponen y no se recapitulan. De esta discusión se originarán puntos de partida para nuevas investigaciones. Se redacta en presente.

Conclusiones: Deberain ser redactadas en forma clara y concisa.

Agradecimiento: Incluirlos solamente si los hubiera, y solo se menciona a quienes contribuyeron con un apoyo muy importante (técnico) 0 las instituciones que han financiado la investigación (si fuera cl caso).

Referencias Bibliográficas: Deben aparecer solamente las referencias bibliográficas utilizadas por el autor en la realización de la investigación y que son mencionadas en la redacción del artículo cientifico. Scrain presentadas en el orden correspondiente al esulo bibliográfico. Considerar que si en el articulo hay quince citas, también debe haber quince referencias bibliográficas.

2.2. Articulo de Revisión: Debe contener: Título en español e inglés; Autor(es); Resumen; Palabras Clave; Abstract; Key Word; Introducción, Método utilizado para localizar y seleccionar los articulos relevantes sobre el tema. Análisis y comparación de los resultados encontrados, coincidencias y discrepancias; conclusiones; recomendaciones; referencias bibliográficas.

2.3. Reporte de Caso Clínico: Debe contener: Titulo en espaniol y en inglés, Autor(es), Resumen, Palabras clave, $\Lambda$ bstract, Key Word, Introducción, Anamnesis, Examen clínico, Exámenes auxiliares (laboratorio y gabinete), Eriología, Diagnóstico preliminar (presuntivo), Tratamiento, Evolución y complicaciones (si las hubiera), Diagnóstico definitivo, Histopatología (si las tuviera), Discusión, Referencias Bibliográficas, Fotografias antes y después del tratamiento. Fin las fotos del rostro de una persona debe cubrirse los ojos para preservar su identidad, para mostrar el rostro completo se debe contar con el conscntimiento escrito, firmado por el paciente y con impresión dactilar de su dedo indicc.

2.4. Articulo de Opinión: Debe contencr: Título en espanol y en inglés; nombre y apellidos del Autor(es), Introducción, Conclusiones, Referencias Bibliográficas.

2.5. Reseña de Libro: De contenido abierto en cuanto al contenido de la reseña. Sin embargo, será obligatorio considerar: Título del libro en mayúsculas, el nombre del autor 0 autores (en minúsculas, excepto las primeras letras del nombre y apellido), la edición, la editorial, el año de publicación, el ISBN (si tuviera) y el número de páginas. Así mismo, la carátula del libro, la editorial (escaneado a colores). El contenido no debe exceder de dos páginas. $A 1$ final de la reseña se debe colocar el nombre del autor de la reseña, indicando además, la licenciatura y los grados académicos (si los tuviera), asi como los principales cargos académicos o institucionales que desempeña.

2.6. Finsayo Científico y Artículo Informativo: I a estructura es: Título en espanool o inglés; nombre y apellidos del Autor(es); Resumen y Palabras Clave; 
Abstract y Key Words; Introducción; Desarrollo Temático; Conclusiones; Referencias Bibliográficas.

2.7. Carta al Editor: Debe contener: Título en español y en inglés, Auror y filiación institucional, Inicio mencionando la razón objetivo de la carta; si fuera necesario, sólo una tabla o una figura; Razón del planteamiento de la opinión, Discusión de resultados y/o recomendaciones, Referencias Bibliográficas, no más de seis referencias biblingráficas, Correo electrónico.

\section{ESTILO DE PRESENTACIÓN}

Los artículos a ser publicados, deberán cumplir los requisitos que a continuación se detalla:

DE LA PRESENTACIÓN: Sc presentará 01 cjemplar impreso de un aproximado de 12 páginas y un CD. Los artículos deber ser preparados en formato MS Word utilizando la fuente Times New Romas de tamaño 10, a espacio sencillo. La primera página del artículo debe incluir; El Título (español e inglés), nombre de autor (es), afiliaciones, abstract y palabras clave. Y utilizarín la misma fuente con distinto tamaño y estilo.

DEL TAMANOY MÁRGENES: En tamaño $\Lambda 4(210 \times 297 \mathrm{~cm})$. Los márgenes deben ser configurados de la siguiente manera: superior e izquierdo: 2.5 $\mathrm{cm}$; inferior $y$ derecho: $2 \mathrm{~cm}$. No se permite agregar pie de página ni encabezados, Las referencias y la bibliografia se agregarán al finalizar el texto

DFI. TITUI.O: El título será escrito en español y en inglés, en tamaño 16 y 14 respectivamente; nombre(s) de autor(es) en tamaño 12 ; la afiliaciỏn o institución/área a la que pertenece, dirección y correo electrônico (tamaño 12). Fl título y autor (es) van en negrita y deben estar centrados.

DEL RESUMEN: Resumen (Abstract) del artículo y palabras clave, español y en inglés. Cada artículo debe incluir un resumen de no más de 200 palabras en la primcra página, seguido por una lista de palabras clave. El resumen debe ser conciso y las palabras clare deben estar justificadas de ambos lados (izquicrdo y derecho)

DEL TEXTO: Estará escrito en dos columnas.

DE LAS REFLRLNCIAS Y CITAS: Utilizar el estilo de referencias bibliográficas acorde a su investigación. El formato de las citas y referencias bibliográficas que se utilizará será: en las ciencias de la salud (con excepción de Psicologia) la norma Vancouver; y en las ciencias sociales, ciencias humanas, ciencias naturales y Psicologia, la norma APA.

DF. I.AS SFCCIONFS: F.l título de una sección debe estar en fuente Times New Roman, tamaño 10 y en negrita, escrito con letras mayúsculas. Debe estar alineado a la izquierda.

DE LAS SU BDIVISIONES: El titulo de las subdivisiones debe cstar alineado a la izquicrda. La fuente a utilizar es Times New Romas tamaño 10 y en negritay sólo las lctras iniciales scrán escritas en mavúscula.

DE LAS FIGURAS O TABLAS: Podrá incluirsc fotografias, gráficos, tablas o imágenes, ctiquetándolos únicamente como figuras o tablas scgún convenga.

Las figuras serán identificadas con la etiqueta Figura, numeradas con números arábigos de manera consecutiva, la cual será ubicada al pie de la figura. Las figuras, deberán adjuntarse adicionalmente como archivo de imagen (jpgo png).

Las tablas serán identificadas con la etiqueta Tabla, numeradas con números arábigos de manera consecutiva y ubicada en la cabecera de la tabla de forma centrada.

Tas figuras y tablas a utilizar deberán ser insertadas en el punto apropiado del texto, debe ser mencionada en el texto al menos una ve\% y antes de su aparición.

DEL IDIOMA, ESTLO Y CONTENIDO: Los artículos deben presentarse en español. El titulo y el resumen están inscritos cn español c inglés. La ortografia y puntuación deben escribirse con estilo sencillo y directo. Utulice estructuras simples para las oraciones, asi como vocabulario común y básico. Defina o explique el vocabulario técnico con sencillez. Lxplique acrónimos cuando aparezcan en el texto por primera vez, La presentación de unidades de medida y valores numéricos se realizará conforme al Sistema Internacional de Unidades, asimismo, debe ucilizarse la coma "," para la separación decimal.

IMPORTAN'TE: Los autores son responsables de asegurarse que su trabajo sea conducido de una manera responsable yética. La estructura y estilo son tan importantes como el contenido. Antes de escribir su artículo, le recomendamos que lea la amplia literatura disponible para informarse respecto a cómo escribir un buen artículotécnico.

\section{PROCESO DE RECIBO, SELECCIÓNYEVALUACIÓN}

4.1. Recepción de los artículos

Las convocatorias para la recepción de artículos se cierran el 30 de abril y 30 de setiembre de cada año. Los articulos se entregan en formato impreso en la Oficina General de Investigación de la Universidad Nacional Jorge Basadre Grohmann sito en Ciudad Úniversitaria Av. Miraflores s/n, Tacna, Perú, o son enviados mediante correo electrónico a la cuenta coin@unjbg.edu.pe.

Junto al artículo (construido en un procesador de textos y guardado en formato doc 0 .docx) se envian las figuras presentadas en él, debidamente identificadas (en formato ipg o pny con alta calidad), los datos de los autores debidamente diligenciados.

F.l formato impreso debe enviarse mediante carta de presentación acompañada del original impreso en papel, más un CD en formato de procesador de textos,

4.2. Proceso de sclccción

Para que un articulo sea admitido en la revista Ciencia \& Desarrollo debe cumplir con los parámetros de forma cstablecidos por la revista.

Los autores que decidan retirar sus articulos del proceso de selección, no implica que pierdan la posibilidad de presentarlo para ediciones futuras.

En los articulos que a pesar de ser aceptados, se detecte algún tipo de plagio no serán admitidos para la impresión final.

Después de un tiempo prudencial, el director del comité editorial comunicará al autor sobre la aceptación de su artículo. 4.3. Proceso de Evaluación

La primera evaluación la realiza el Comité Editorial, sobre el cumplimiento de los requisitos formales y la adecuación al interés temático de la Revista, de la cual se envía un mensaje a los autores donde se señala su resultado. Fin caso de corresponder al interés de la revista, se indican a los autores que aquellos aspectos a ajustary la fecha limite para hacer la nueva entrega.

Comprobado el cumplimiento de los requisitos formales, cl articulo cs cnviado a cvaluación por parte de arbitros expertos, intcgrantes del Comité Cientifico de la revista. Este arbitrajc sc hace de forma confidencial (doble ciego; los árbitros desconocen los nombres de los autores y viceversa), mediante cl diligenciamiento de un formato específico, que solicita su concepto sobre la conveniencia o no de su publicación, y las recomendaciones para mejoramiento del articulo. El resultado de la evaluación se informa a los autores oportunamente mediante correo electrónico, y en caso de requerirse, se indica la fecha para la entrega de los documentos ajustados, con el fin de que los árbitros emitan su concepto definitivo sobre la condición de publicable o no. 